\title{
Article \\ Impact of Charge-Resonance Excitations on CT-Mediated J-Type Aggregation in Singlet and Triplet Exciton States of Perylene Di-Imide Aggregates: A TDDFT Investigation
}

\author{
Yasi Dai ${ }^{1}(\mathbb{D})$, Maria Zubiria-Ulacia ${ }^{2,3}$ (D), David Casanova ${ }^{2,4}(\mathbb{D})$ and Fabrizia Negri $1,5, *(\mathbb{D})$ \\ 1 Dipartimento di Chimica “Giacomo Ciamician", Università di Bologna, 40126 Bologna, Italy; \\ yasi.dai2@unibo.it \\ 2 Donostia International Physics Center (DIPC), 20018 Donostia, Euskadi, Spain; \\ maria.zubiria@dipc.org (M.Z.-U.); david.casanova@ehu.eus (D.C.) \\ 3 Universidad del Pais Vasco/Euskal Herriko Unibertsitatea, Manuel Lardizabal 3, 20018 Donostia, Euskadi, Spain \\ 4 IKERBASQUE, Basque Foundation for Science, 48009 Bilbao, Euskadi, Spain \\ 5 INSTM, UdR Bologna, 40126 Bologna, Italy \\ * Correspondence: fabrizia.negri@unibo.it
}

check for updates

Citation: Dai, Y.; Zubiria-Ulacia, M.; Casanova, D.; Negri, F. Impact of Charge-Resonance Excitations on CT-Mediated J-Type Aggregation in Singlet and Triplet Exciton States of Perylene Di-Imide Aggregates: A TDDFT Investigation. Computation 2022, 10, 18. https://doi.org/ 10.3390/computation 10020018

Academic Editor: Henry Chermette

Received: 5 January 2022

Accepted: 20 January 2022

Published: 25 January 2022

Publisher's Note: MDPI stays neutral with regard to jurisdictional claims in published maps and institutional affiliations.

Copyright: () 2022 by the authors. Licensee MDPI, Basel, Switzerland. This article is an open access article distributed under the terms and conditions of the Creative Commons Attribution (CC BY) license (https:// creativecommons.org/licenses/by/ $4.0 /)$.

\begin{abstract}
The modulation of intermolecular interactions upon aggregation induces changes in excited state properties of organic molecules that can be detrimental for some optoelectronic applications but can be exploited for others. The time-dependent density functional theory (TDDFT) is a costeffective approach to determining the exciton states of molecular aggregates, and it has been shown to provide reliable results when coupled with the appropriate choice of the functional. Here we apply a general procedure to analyze the aggregates' exciton states derived from TDDFT calculations in terms of diabatic states chosen to coincide with local (LE) and charge-transfer (CT) excitations within a restricted orbital space. We apply the approach to study energy profiles, interstate couplings, and the charge-transfer character of singlet and triplet exciton states of perylene di-imide aggregates (PDI). We focus on the intermolecular displacement along the longitudinal translation coordinate, which mimics different amounts of slip-stacking observed in PDI crystals. The analysis, in terms of symmetry-adapted Frenkel excitations (FE) and charge-resonance (CR) states and their interactions, discloses how the interchange of the $\mathrm{H} / \mathrm{J}$ character for small longitudinal shifts, previously reported for singlet exciton states, also occurs for triplet excitons.
\end{abstract}

Keywords: molecular aggregates; singlet excitons; triplet excitons; TDDFT; charge-transfer states; charge-resonance states; Frenkel states; localized excitations; diabatic states; adiabatic states

\section{Introduction}

The photophysical behavior of organic electronic molecular materials is governed by the nature of their low-lying exciton states [1-17]. Exciton states are superpositions of local excitations (LEs) and charge-transfer (CT) states, namely diabatic states describing electron promotions between occupied molecular orbitals to unoccupied molecular orbitals of the same (neutral) or neighboring (ionic) molecules (or sites), respectively [18-32].

The role of CT states on photo-induced processes is documented by a large number of investigations. For instance, CT states might facilitate intersystem crossing through spin-orbit coupling [33] and are often involved in the first step of singlet fission [34-40] through the process of symmetry-breaking charge separation (SBCS). CT states also have a crucial role in exciton dissociation and charge separation in heterojunctions between electron-donating and electron-accepting materials or in homojunctions between crystalline domains with different orientations [41-43].

While the modelling and analysis of singlet spin exciton states has received considerable attention $[20,22,27,44-50]$, triplet excitons have received comparably less attention 
and only few investigations have been reported [22,51,52]. A relevant role of triplet states in photoinduced processes is, however, proven and supported by experiments. Enhanced triplet-state generation, following photo-induced charge transfer, was reported by various groups in electron donor-acceptor polymer-blend films in organic photovoltaic devices where nonemissive triplet excitons are responsible for nonradiative-charge recombination [53]. Thus, triplet formation by charge recombination can be a detrimental, unwanted side effect but can also be exploited as a useful triplet-generation method, for instance in photocatalysis and photodynamic therapy $[54,55]$. Examples are, among others, triplet-state generation in molecular dyads [56] and molecular dimers in which dimerization-induced triplet population has been invoked [57].

A deep insight into the relationship between structural arrangement and triplet excitonstate character is, therefore, essential to identify molecular systems and condensed phase organizations that are most suitable to generate and exploit triplet exciton states.

Perylene-bis(dicarboximide) (PDI) and its derivatives have attracted great interest as chromophores for energy and charge-transport studies, thanks to their propensity to self-organize into ordered assemblies, both in solution and in the solid state via $\pi-\pi$ stacking interactions $[11,12,58]$. Numerous computational investigations on PDI aggregates have been focused on the prediction of exciton states with different quantum-chemical (QC) approaches, including configuration interaction truncated to single excitations (CIS), time-dependent density functional theory (TDDFT) $[22,27,46,47,59,60]$, and highly accurate levels of theory [61-63].

TDDFT is a cost-effective approach to evaluating exciton states of molecular aggregates and has been shown to provide reliable results when coupled to a suitable choice of the exchange-correlation functional. To analyze the character of excitonic states predicted by QC calculations, a diabatization procedure can be used to determine the superposition of LE and CT diabatic states in each adiabatic exciton state [21,22,31,32,59].

For aggregates characterized by a symmetric arrangement of chromophores, as those investigated here (Figure 1), when the molecules approach each other, intermolecular interactions mix the LE states to form a symmetry-adapted (SA) superposition of (neutral) LE states, that is, Frenkel excitons (FE). Similarly, the ionic states form delocalized chargeresonance (CR) states $[18,64,65]$ of appropriate symmetry. The symmetry point group of a PDI aggregate, where intermolecular displacements along the longitudinal translation coordinate, $z$, are considered, (Figure 1 ) is $C_{2 h}$. As a result, the most relevant $\pi \pi^{*}$ exciton states, along with the FE and CR diabatic states, all belong to $A_{g}$ and $B_{u}$ symmetry representations. Symmetry can be exploited to easily identify $\mathrm{H}$ - and J-aggregation types, since only $B_{u}$ exciton states are accessible as dipole-allowed transitions.

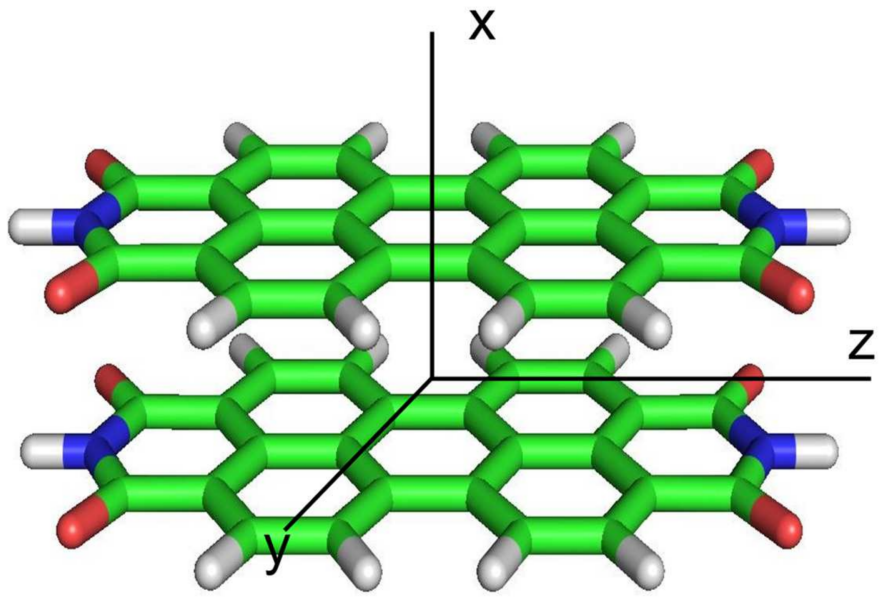

Figure 1. The PDI dimer considered in this work. Singlet and triplet exciton states have been determined at the eclipsed configuration shown here and along the interchromophore longitudinal (z-axis) translation coordinate. 
Here we adopt a similar strategy to characterize singlet and triplet exciton states of PDI aggregates. To this end, we analyze exciton states in terms of LE and CT contributions, along the lines described in ref. [60], with three major objectives. First, we validate the protocol of ref. [60] by comparing the computed exciton states character (for both singlet and triplet exciton states) with the results obtained from the character-analysis tool, TheoDORE [66]. Second, we show that for triplet exciton states the modulation of CR/FE interactions along the longitudinal translation coordinate determines a switch in the symmetry $\left(A_{g} / B_{u}\right)$ of the lowest triplet exciton state, which corresponds to the unconventional $\mathrm{H}$-/J-character alternation previously documented for singlet excitons $[8,9,60]$. Finally, we demonstrate that the selection of the functional is less critical for triplet excitons compared to singlet excitons and show that the CAM-B3LYP and $\omega$ B97X-D results are very close to each other.

\section{Computational Methods}

The protocol employed to analyze the character of exciton states transforms TDDFT amplitudes from the basis of single excitations between the aggregate's orbitals (the delocalized excitation (DE) basis) to the basis of single excitations between molecular site orbitals, the latter defining the diabatic states. It has been previously described [60] and is briefly summarized here. To analyze the exciton character, we express each relevant exciton state in terms of LEs and CTs. To this end, we select the orbital subspace corresponding to relevant $\pi \pi^{*}$ exciton states. For PDI aggregates this includes the HOMO and LUMO of each monomer $[21,31,59,60,67]$ and represents the minimal orbital space (MIOS). The next step involves the determination of the linear combination coefficients, $C_{i, j}^{A G G R} R_{-} M O B$, forming the $C_{A G G R \_M O B}$ matrix and describing each aggregate's orbital in the monomer orbital basis (MOB) as [60,68,69]:

$$
C_{A G G R \_M O B}=C_{M O N_{-} A O B}^{t} \cdot S_{M O N_{-} A O B} \cdot C_{A G G R \_A O B}
$$

where the $C_{M O N_{-} A O B}$ matrix is a block diagonal matrix containing the molecular orbitals' (MOs) coefficients in the atomic orbital basis (AOB) of each monomer, with off-diagonal blocks set to zero, and $S_{M O N_{-} A O B}$ is the overlap matrix of the monomers in the AOB.

In general, monomer orbitals belonging to two different molecules are nonorthogonal to each other. Hence, the aggregate's orbitals, $C_{A G G R \_M O B}^{L}$, expressed in terms of orthogonalized monomer orbitals are obtained as:

$$
C_{A G G R \_M O B}^{L}=S_{A G G R \_M O B}^{-\frac{1}{2}} \cdot C_{A G G R \_M O B}
$$

where superscript $L$ indicates Löwdin's orthogonalization [70], and the overlap matrix $S_{A G G R \_M O B}=C_{M O N_{-} A O B}^{t} \cdot S_{A G G R_{-} A O B} \cdot C_{M O N_{-} A O B}$ is obtained from the coefficients of the monomer's orbitals, $C_{M O N_{-} A O B}$, and the overlap of the atomic orbitals in the aggregate configuration, $S_{A G G R \_A O B}$.

From the results of the TDDFT calculations on the aggregate, the subset of the $n^{2}$ exciton states originated from the MIOS are then selected out of the full set of computed eigenstates. TDDFT amplitudes are expressed based on the DEs, namely excitations between the aggregate's orbitals, and form the columns of the $\boldsymbol{B}_{D E}^{\text {adia }}$ matrix. Thus, the following step is required to expand each DE in terms of diabatic LE and CT states (excitations between monomer orbitals). With the aggregate's orbitals expressed in terms of monomer orbitals via the $C_{A G G R \_M O B}^{L}$ matrix, each excitation from an occupied $i$ to an empty $j$ aggregate's orbital $\mathrm{DE}(i \rightarrow j)$ can be expressed as a linear combination of diabatic (LE and CT) excitations $(k \rightarrow l)$ from an occupied $k$ to an empty $l$ monomer orbital, with the expansion coefficients forming the columns of the unitary matrix $U_{D E \rightarrow \text { dia }}$ given by

$$
U_{k \rightarrow l, i \rightarrow j}^{D E \rightarrow \text { dia }}=C_{k, i}^{A G G R \_M O B, L} \cdot C_{l, j}^{A G G R_{-} M O B, L}
$$

Exciton states are then readily expressed in the diabatic basis as $B_{\text {dia }}^{\text {adia }}=U_{D E \rightarrow \text { dia }} \cdot \boldsymbol{B}_{D E}^{\text {adia }}$, and their character is obtained by summing up the contributions from the CT and LE states. 
The corresponding $n^{2}$ eigenvalues (excitation energies of the selected excitons) form the diagonal $\boldsymbol{H}_{\text {adia }}$ matrix, from which the Hamiltonian in the diabatic LE/CT basis, $\boldsymbol{H}_{\text {dia }}$ can be obtained as $[21,30,32,71]$

$$
\boldsymbol{H}_{\text {dia }}=\boldsymbol{B}_{\text {dia }}^{\text {adia }} \cdot \boldsymbol{H}_{\text {adia }} \cdot \boldsymbol{B}_{\text {dia }}^{\text {adiat }}
$$

Finally, the $\boldsymbol{H}_{\text {dia }}$ is rotated in the SA diabatic basis formed by FE and CR excitations to obtain two matrices, $\boldsymbol{H}_{\text {dia }}^{\text {sym }}$ (one for $B_{u}$ states and one for $A_{g}$ states), whose off-diagonal elements are the interactions between the CR and FE states that ultimately govern the modulation of adiabatic exciton-state energies along the longitudinal translation coordinate. These interaction energies have been shown to correspond to \pm combinations of electron$\left(D_{e}\right)$ and hole- $\left(D_{h}\right)$ transfer integrals $[22,31,59,60]$.

The PDI-monomer structure was the same one used in previous investigations on PDI aggregates, optimized at the BLYP-D/TZV(P) level of theory [72]. The distance between the planes of different monomers was set to $3.4 \AA$, which is a distance used in previous investigations on dimers of PDI $[59,60]$. Exciton states were computed for the eclipsed aggregates and for displacements of $0.5 \AA$ up to $8.0 \AA$ along the longitudinal translation coordinate (z) (Figure 1). For singlet exciton states, trimers and tetramers were also considered beside dimers (Figure S1).

Excitation energies were determined with TDDFT calculations in the Tamm-Dancoff approximation (TDA) [73], using the CAM-B3LYP [74] and $\omega$ B97X-D [75] functionals and the $6-31 \mathrm{G}^{*}$ basis set. The $\omega \mathrm{B} 97 \mathrm{X}-\mathrm{D}$ functional was previously shown to provide a reliable description of CT character in singlet excitons of PDI dimers [27]. All QC calculations were carried out with the Gaussian16 suite of programs [76]. The CT character of exciton states was determined as described above and using TheoDORE 2.4 [66].

\section{Results and Discussion}

\subsection{Modulation of Singlet Exciton-State Energies and Characters for PDI Aggregates}

Previous investigations of excitation energy profiles and CT character in PDI dimers for interchromophore longitudinal shifts have shown that computed exciton states are generally strong mixtures of the two types of diabatic states. The weight of LE and CT characters, however, strongly depends on the energy difference between FE and CR diabatic states, and on their couplings $[27,59,60]$. This is the reason why the CT character of the lowest singlet exciton state is critically dependent on the chosen functional. In this regard, it has been shown that the adiabatic energy profiles and CT characters computed at the TD- $\omega$ B97X-D / 6-31G* level match those obtained at higher level of theory $[27,59,60]$. Here we performed the character analysis based on TDA-computed exciton states rather than the full TDDFT linear response. While the excitation energy profiles of the four exciton states are quite similar (Figure S2), the FE and CT energy profiles of $B_{u}$ symmetry obtained from TDA- $\omega$ B97X-D/6-31G* calculations display a crossing (Figure 2) not seen for TD- $\omega$ B97X$\mathrm{D} / 6-31 \mathrm{G}^{*}$ calculations [60], leading ultimately to a CT character of the lowest $B_{u}$ exciton state (at the eclipsed geometry and for small displacements, see Figure 3 ) greater than $50 \%$, since the weight of the CT character can be traced back to the energy location of FE and CR states [60] (Figure S3). Such large CT character contrasts with experimental results and was previously reported for other long-range corrected functionals and from CIS calculations [27,59]. Obviously, slightly larger intermonomer separations will reduce the contrasting results between TD and TDA- $\omega$ B97X-D/6-31G* calculations, which, however, illustrate the crucial role determined by the energy difference between the $\mathrm{CR}$ and FE states. 


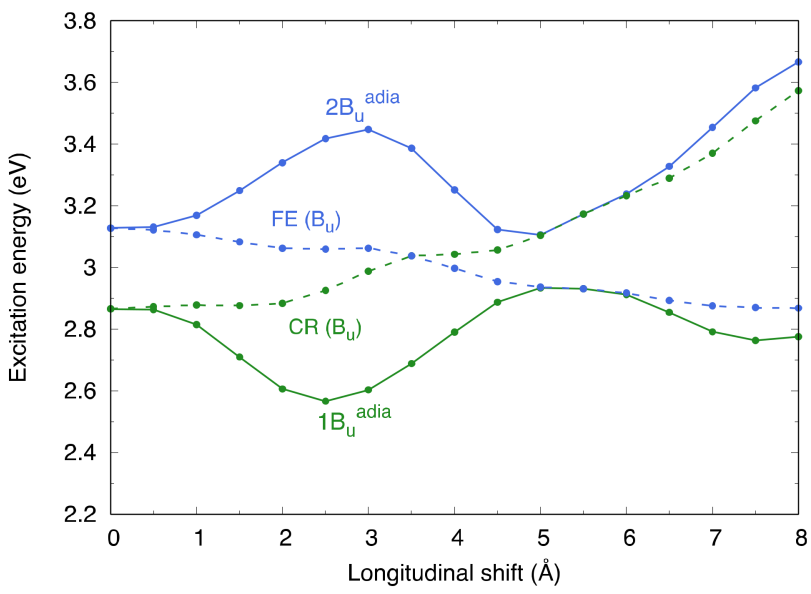

Figure 2. Excitation energy profiles (TDA- $\omega$ B97X-D/6-31G*) of $B_{u}$ adiabatic (adia, solid) singlet exciton states and $B_{u} \mathrm{FE}$ and $\mathrm{CR}$ states (dashed) for the PDI dimer along the longitudinal translation coordinate. The computed energy profiles of exciton states are the result of the interactions between $\mathrm{CR}$ and FE states of the same symmetry. When the interactions are large, the adiabatic exciton energies differ considerably from the diabatic energies.

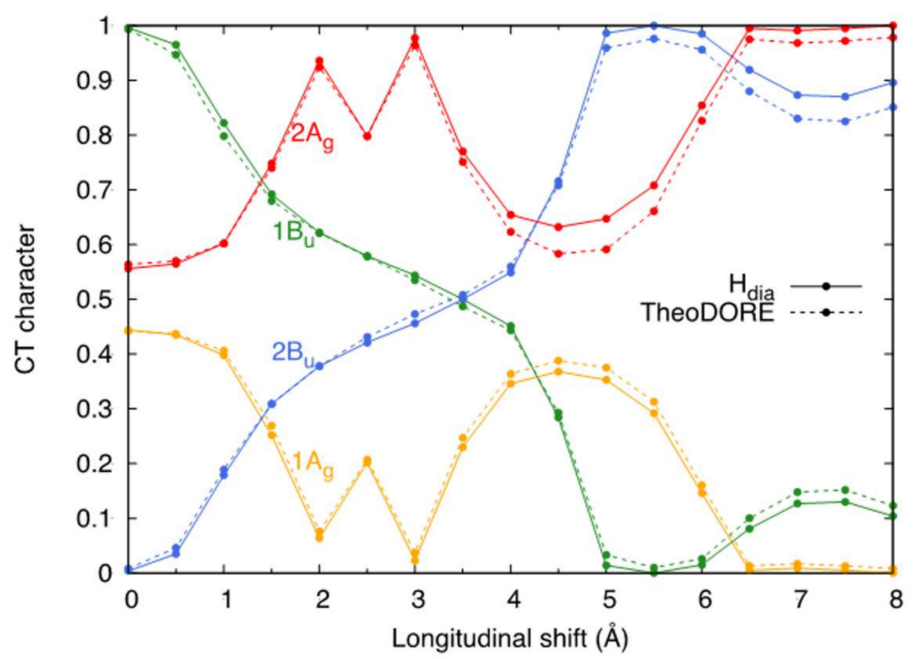

Figure 3. CT character of adiabatic singlet exciton states of PDI dimer along the longitudinal translation coordinate determined with two different approaches (solid) using the procedure described in Section 2 and (dashed) using TheoDORE analysis tool [66].

Finally, we note that the CT character determined following the procedure outlined in Section 2 and the results of TheoDORE [66] are in excellent agreement for all the four low-lying states investigated (Figure 3). A similar correspondence is found for the lowest energy exciton states of larger aggregates (Figure S4).

\subsection{Modulation of Triplet Exciton-State Energies and Characters for PDI Dimers}

Triplet exciton-state analysis was carried out only on TDA calculations since TD triplet wavefunctions contain relevant contributions from de-excitations. The oscillating trend in adiabatic energy profiles (Figure 4a) are easily rationalized by the oscillating trend of the interactions between the CR and FE states (Figure 4b). As shown previously $[22,31,59,60]$, these interactions are given by the \pm combinations of the $D_{e}$ and $D_{h}$ transfer integrals. While couplings between triplet LE states are almost negligible [22], spin triplet CR/FE interactions are of the same magnitude as those computed for singlet exciton states (Figure S5). In analogy with singlet excitons, these interactions determine not only the CT character of the adiabatic triplet exciton states (Figure 4c), but also the symmetry of the lowest energy 
state (Figure $4 \mathrm{a}$ ), with an interchange of $A_{g}$ and $B_{u}$ along the longitudinal translation coordinate. Such an alternation is well established for singlet exciton states and leads to the appearance of CT-mediated J-type spectroscopic features for small longitudinal displacements of one of the two PDI molecules $[8,9,58,60]$ in a range of displacements where Kasha's theory only predicts H-type aggregation. Therefore, CR states create an effective short-range exciton coupling that can induce unconventional J-type spectroscopic features $[8,9,46,58-60,69,77,78]$.
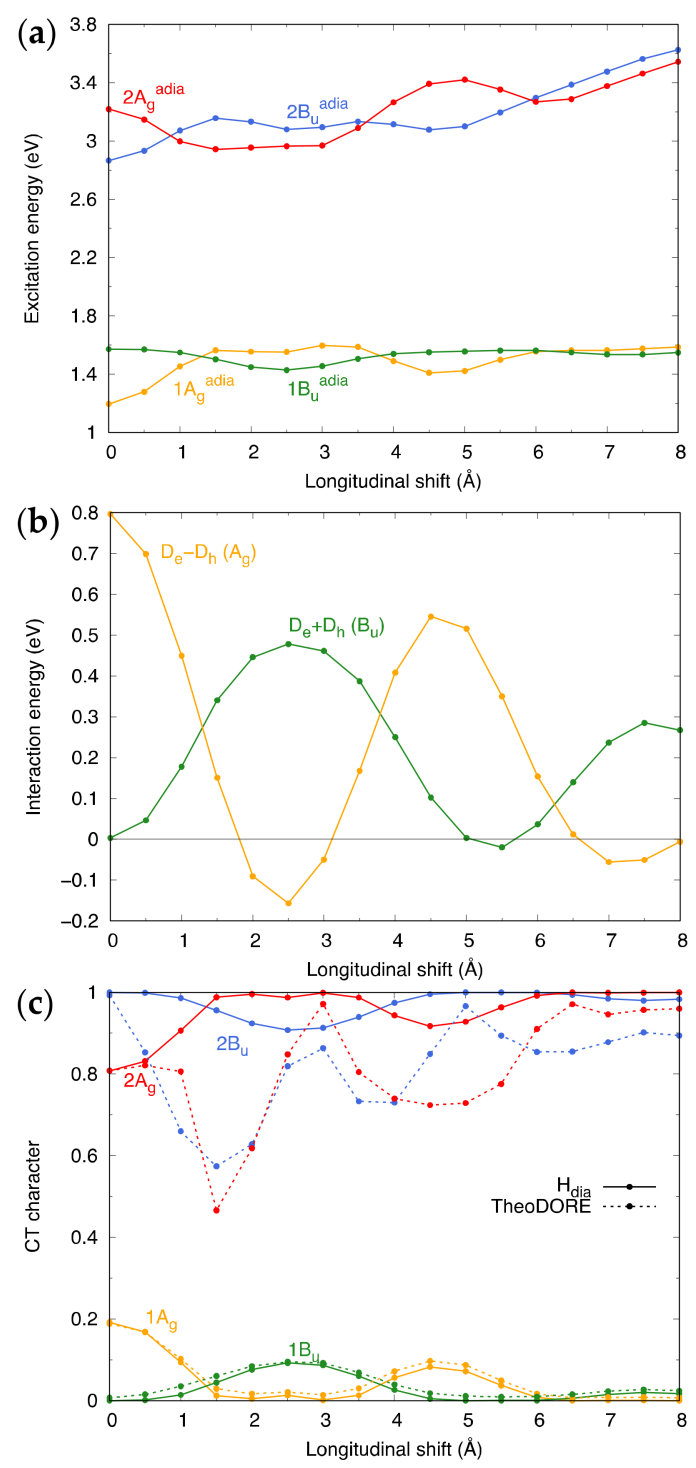

Figure 4. Analysis of triplet exciton states of the PDI dimer (TDA- $w$ B97X-D/6-31G*). (a) Computed adiabatic excitation energy profiles. (b) Magnitude and modulation along the longitudinal translation coordinate of the $D_{e} \pm D_{h}$ terms for the interaction between CR/FE states of the dimer. (c) Comparison between the CT character determined with the procedure described in Section 2 and with TheoDORE [66] for the selected triplet exciton states of PDI dimer.

Notably, here we show that the same mechanism is active in the triplet exciton manifold, albeit with less effective CR/FE mixing. Indeed, as previously reported [22], one of the most relevant differences between singlet and triplet exciton states is the larger energy difference between FE and CR states, which triggers a modest CR/FE mixing. This can be appreciated in Figure 4c, showing that the largest CT contribution for the lowest triplet exciton state does not exceed $20 \%$. At the same time, Figure 5a,b compare the results of $\mathrm{CR} / \mathrm{FE}$ interactions for singlet and triplet $A_{g}$ exciton states of the PDI dimer and enlighten 
the role of the increased energy difference between FE and CR states in the latter. Nevertheless, the CR/FE mixing is sufficient to modulate the triplet exciton energies, ultimately leading to an alternation between $B_{u}$ and $A_{g}$ states that parallels what is well documented for singlet exciton states.

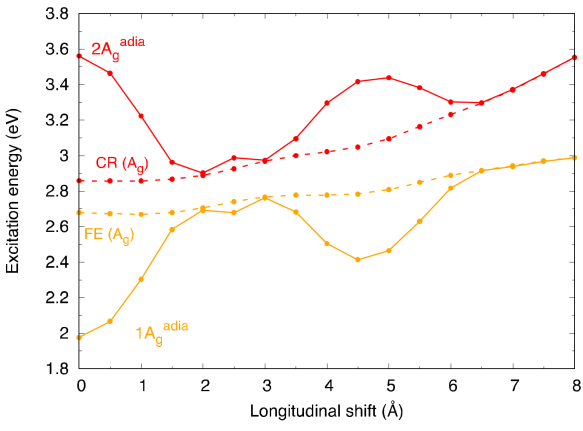

(a)

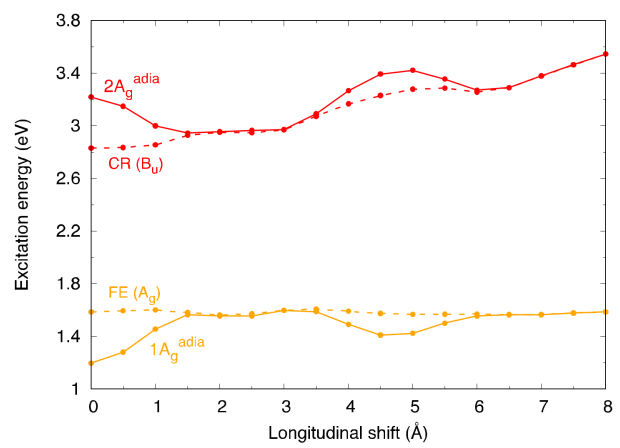

(b)

Figure 5. Energy profiles (TDA- $\omega$ B97X-D/6-31G*) of (solid) $A_{g}$ adiabatic exciton states and (dashed) $A_{g}$ FE and CR states for the PDI dimer along the longitudinal translation coordinate: (a) singlet exciton states; (b) triplet exciton states. The computed exciton energy profiles result from the interactions between CR and FE states of the same symmetry. When the interactions (Figure $4 \mathrm{~b}$ ) are strong, the exciton-state energies deviate from FE and CR energies. The interaction is less effective for triplet states due to the larger energy difference between FE and CR states.

More specifically, the oscillation of the CR/FE interaction (see the $D_{e} \pm D_{h}$ profiles shown in Figure $4 \mathrm{~b}$ ), determines an interchange of the lowest exciton states of $A_{g}$ and $B_{u}$ symmetry in the dimer for longitudinal shifts in the range of ca. 2-3 $\AA$, where the interaction between $A_{g}$ symmetry FE and CR states is almost negligible. At the same time, the interaction between the FE and $\mathrm{CR} B_{u}$ states is the maximum and pushes the adiabatic $1 B_{u}$ state energetically below $1 A_{g}$ (see Figure $4 \mathrm{a}$ ), thereby switching the aggregate character from $\mathrm{H}$ - to J-type.

These results suggest that radiative and nonradiative decays from the lowest triplet exciton states of molecular aggregates, intimately related to the nature and symmetry of the lowest energy state, may be modulated by the intermolecular organization, a concept that could be exploited for systems displaying dimerization-induced triplet state populations [57].

We finally compare the CT character analysis obtained from our protocol (solid lines in Figure 4c) with the results of TheoDORE (dashed lines in Figure 4c). The agreement is very good for the two lowest triplet exciton states, while marked differences appear for the remaining two states. The reason is that for several intermolecular configurations the selected $\pi \pi^{*}$ triplet exciton states (whose wavefunction is dominated by excitations within the selected $\pi \pi^{*}$ orbital space) are spread over more than one TDDFT-computed exciton state. As a consequence, TheoDORE analysis picks up only the CT contribution corresponding to the fraction of the selected $\pi \pi^{*}$ exciton state. In contrast, with the procedure described in Section 2, the selected $\pi \pi^{*}$ exciton states are projected out of the entire space of computed exciton states and renormalized. Interestingly, triplet exciton states (belonging to the selected orbital space) appear to mix more strongly than singlet states with other exciton states of the dimer, as suggested by wavefunction analysis and by the fragmentation of the CT contributions in TheoDORE analysis. Such fragmentation contrasts with the almost perfect match discussed for singlet excitons of the PDI dimer (Figure 2) and suggests that diabatization procedures are likely to be more problematic when analyzing triplet exciton states.

To visualize the nature of exciton states for two representative dimer configurations of the PDI dimer, we have carried out a fragment-based analysis via electron-hole correlation plots using TheoDORE (Figure 6). The two selected fragments correspond to the two molecules forming the dimer. Exciton states are identified by the nonvanishing elements of 
the $2 \times 2$ matrix (the $\Omega$-matrix [66]) represented by different levels of grey. Locally excited contributions appear on the main diagonal (going from lower left to upper right), while CT contributions appear off diagonally. In agreement with the character analysis shown in Figure $4 c$, Figure 6 shows that for the eclipsed configuration, the character of the $1 B_{u}$ state is purely LE while the character of the lowest energy exciton state of $1 A_{g}$ is partially CT, as indicated by the light grey squares in the electron-hole correlation plot. The situation is reversed at the slip-stacked configuration (2.5 $\AA$ longitudinal translation), with the $1 B_{u}$ state displaying some CT contribution.

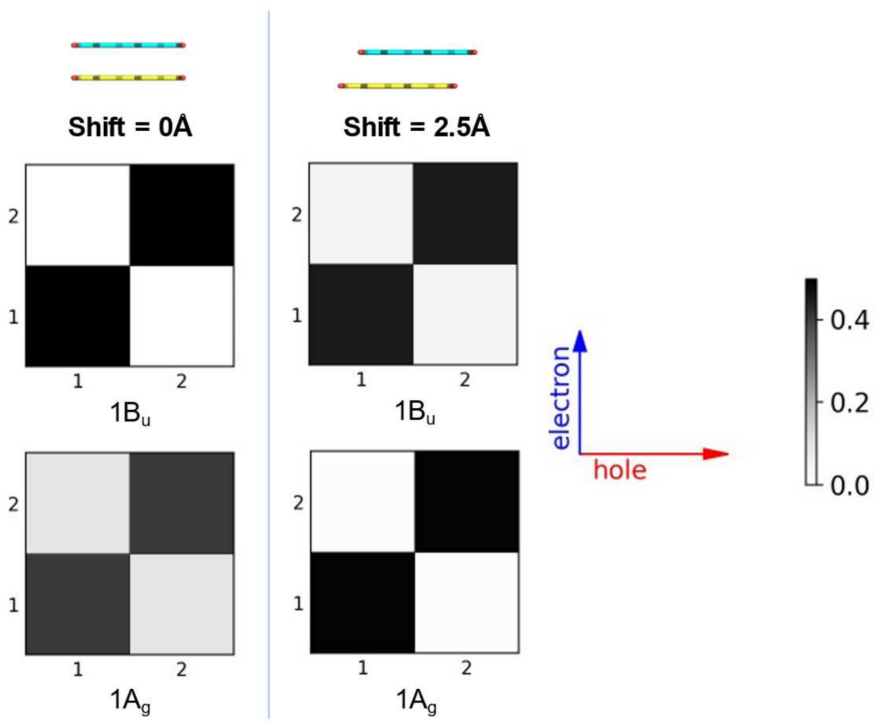

Figure 6. Triplet exciton-state analysis via electron-hole correlation plots [66] for two PDI dimer configurations: eclipsed $0.0 \AA$ and $2.5 \AA$ slip-stacked. The grey scale used is shown on the right panel. From TDA- $\omega B 97 X-D / 6-31 G^{*}$ calculations.

Influence of Functional on Triplet Exciton Character of the PDI Dimer

While the interplay of CR and FE states is crucial for TDDFT functionals to reproduce higher level results for the calculation of singlet excitons $[27,60]$, the larger energy difference between FE and CR triplet states makes the choice of the functional less critical. This is illustrated by the small differences obtained between TDA- $\omega$ B97X-D/6-31G* and TDACAM-B3LYP/6-31G* calculations.

The major difference between the two functionals is the lower excitation energies computed at the TDA-CAM-B3LYP level. The difference between the TDA-CAM-B3LYP and TDA- $\omega$ B97X-D energies is larger for the two higher energy exciton states, whose CT character is dominant (Figure 7). The lower energy of CT states at the TD-CAM-B3LYP level is not unexpected and was identified as the major source of discrepancy for singlet exciton states computed with the CAM-B3LYP functional compared to $\omega$ B97X-D [27]. As a result of the lower energy of CT states with the CAM-B3LYP functional, the CT character of the lowest energy triplet exciton states is slightly larger at the TDA-CAM-B3LYP level, compared to TDA- $\omega B$ 97X-D, as can be seen in Figure 8. Interestingly, the CR/FE interaction is virtually independent of the chosen functional, with almost identical TD-CAM-B3LYP and TDA-CAMB3LYP values (Figure 9). While it can be expected that other long-range corrected hybrid variants would provide results similar to those reported here, the use of nonhybrid or hybrid functionals with small contributions of exact (HF) exchange is not recommended because of their tendency to underestimate the energetic position of CT states [46]. 


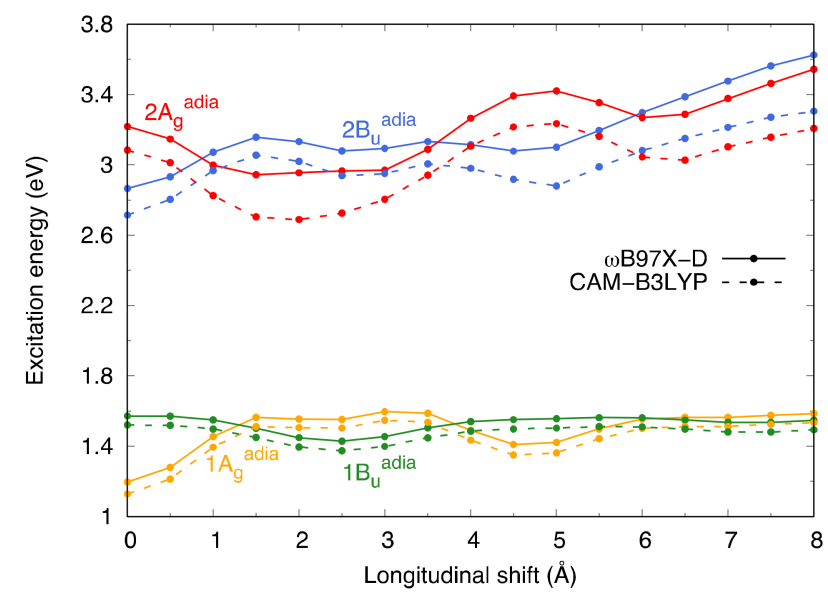

Figure 7. Comparison between excitation energies of triplet exciton states of PDI dimer computed with TDA-CAM-B3LYP /6-31G* (dashed) and TDA- $\omega$ B97X-D/6-31G* (solid) along the longitudinal shift.

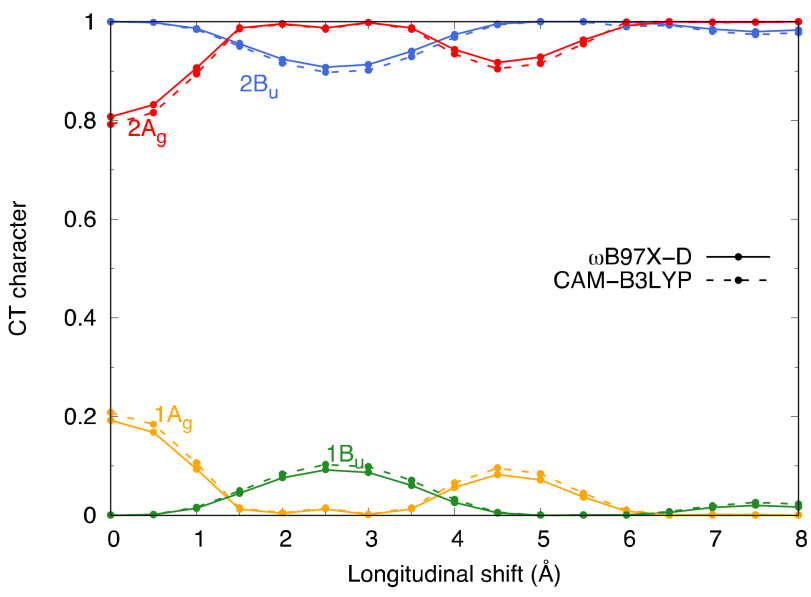

Figure 8. Comparison between triplet exciton CT character computed with TDA-CAM-B3LYP/6-31G* (dashed) and TDA- $\omega$ B97X-D/6-31G* (solid) calculations.

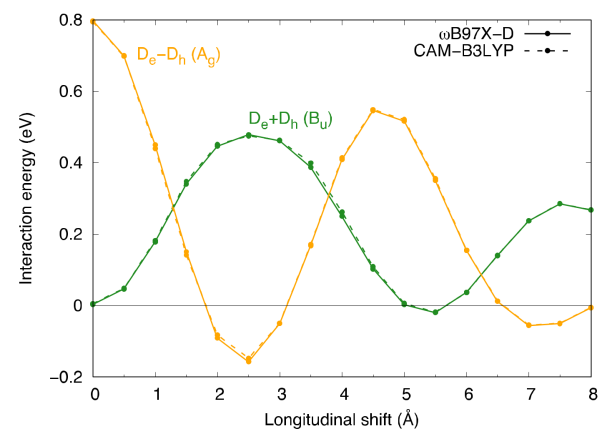

Figure 9. Interaction energies between CR and FE states computed at TDA-CAM-B3LYP/6-31G* (dashed) and TDA- $\omega$ B97X-D/6-31G* (solid) levels; (gold) interaction between $A_{g}$ CR and FE states; (green) interaction between $B_{u} \mathrm{CR}$ and FE states.

\section{Conclusions}

We have analyzed the modulation of singlet and triplet exciton states of PDI aggregates computed at the TDDFT level, focusing on the intermolecular displacement along the longitudinal translation coordinate, which mimics different amounts of slip stacking observed in PDI crystals. 
The CT character of singlet and triplet exciton states has been determined with a diabatization procedure, and it has been shown that the results agree with other characteranalysis tools, such as the TheoDORE software.

The study has shown that triplet exciton-state energies can be rationalized in terms of the interactions between CR and FE diabatic states, which are found to be of the same strength as those computed for singlet exciton states. Such CR/FE interactions ultimately lead, not only to a mixed LE/CT character of the low-lying triplet exciton states, but also to a CT-mediated J-aggregation mechanism for small longitudinal displacements, similar to what has been previously documented for singlet exciton states.

Finally, we have compared the results of two long-range corrected hybrid functionals and shown that the magnitude of the $\mathrm{CR} / \mathrm{FE}$ interactions are almost independent on the selected functional. In contrast with singlet exciton states, the larger energy difference between FE and CR triplet states makes the choice of the functional (among long-range corrected hybrid variants) less critical to define the CT contributions in low-lying triplet exciton states.

Overall, these results demonstrate that the same interactions responsible for the CTmediated J-aggregation in singlet exciton states of PDI aggregates are operative in triplet exciton states and show that the role of CR states must be carefully considered as a factor influencing the processes following triplet exciton generation. These findings might be exploited to design chromophores and interchromophore organizations to optimize dimerinduced triplet state population.

Supplementary Materials: The following supporting information can be downloaded at: https: / / www.mdpi.com/article/10.3390/computation10020018/s1, Figure S1:The PDI trimer and tetramer considered in this work; Figure S2:Comparison between adiabatic energy profiles of the four singlet exciton states of PDI dimer along the longitudinal shift calculated at TDA- $\omega$ B97X-D/6-31G* (dashed) and TD- $\omega B$ 97X-D/6-31G* (solid) levels; Figure S3:Comparison between FE and CR singlet states of Bu symmetry calculated at TDA- $\omega$ B97X-D (dashed) and TD- $\omega$ B97X-D (solid) levels. 6-31G* basis set is used; Figure S4:CT character of adiabatic singlet exciton states of PDI trimer $(a, b)$ and tetramer $(\mathrm{c}, \mathrm{d})$ along the longitudinal translation coordinate determined with two different approaches; Figure S5:Comparison between the modulation of the $D_{e} \pm D_{h}$ terms of singlet (dashed) and triplet (solid) exciton states of the dimer along the longitudinal translation coordinate.

Author Contributions: Conceptualization, F.N.; investigation and formal analysis, F.N., D.C., Y.D., and M.Z.-U.; writing—original draft preparation, F.N.; writing—review and editing, F.N., D.C., and Y.D. All authors have read and agreed to the published version of the manuscript.

Funding: This research was funded by Ministerio de Economía y Competitividad of Spain, grant number PID2019-109555GB-I00 and RED2018-102815-T and by Eusko Jaurlaritza, grant number PIBA19-0004.

Institutional Review Board Statement: Not applicable.

Informed Consent Statement: Not applicable.

Data Availability Statement: The data presented in this study are available in the Supplementary Material.

Acknowledgments: The authors acknowledge financial support from the Ministerio de Economía y Competitividad of Spain, from the Eusko Jaurlaritza, and from "Valutazione della Ricerca di Ateneo" (VRA)-University of Bologna, Italy. Y.D. acknowledges Ministero dell'Università e della Ricerca (MUR), Italy, for her PhD fellowship.

Conflicts of Interest: The authors declare no conflict of interest.

\section{References}

1. Ostroverkhova, O. Organic Optoelectronic Materials: Mechanisms and Applications. Chem. Rev. 2016, 116, 13279-13412. [CrossRef] [PubMed]

2. $\mathrm{Xu}, \mathrm{Y} . ; \mathrm{Xu}, \mathrm{P} . ; \mathrm{Hu}, \mathrm{D} . ; \mathrm{Ma}, \mathrm{Y}$. Recent progress in hot exciton materials for organic light-emitting diodes. Chem. Soc. Rev. 2021, 50, 1030-1069. [CrossRef] [PubMed]

3. Samanta, S.; Chaudhuri, D. Suppressing Excimers in H-Aggregates of Perylene Bisimide Folda-Dimer: Role of Dimer Conformation and Competing Assembly Pathways. J. Phys. Chem. Lett. 2017, 8, 3427-3432. [CrossRef] 
4. Hong, Y.; Kim, J.; Kim, W.; Kaufmann, C.; Kim, H.; Würthner, F.; Kim, D. Efficient Multiexciton State Generation in ChargeTransfer-Coupled Perylene Bisimide Dimers via Structural Control. J. Am. Chem. Soc. 2020, 142, 7845-7857. [CrossRef] [PubMed]

5. Haldar, R.; Mazel, A.; Krstić, M.; Zhang, Q.; Jakoby, M.; Howard, I.A.; Richards, B.S.; Jung, N.; Jacquemin, D.; Diring, S.; et al. A de novo strategy for predictive crystal engineering to tune excitonic coupling. Nat. Commun. 2019, 10, 2048. [CrossRef] [PubMed]

6. Haldar, R.; Mazel, A.; Joseph, R.; Adams, M.; Howard, I.A.; Richards, B.S.; Tsotsalas, M.; Redel, E.; Diring, S.; Odobel, F.; et al Excitonically Coupled States in Crystalline Coordination Networks. Chem.-Eur. J. 2017, 23, 14316-14322. [CrossRef] [PubMed]

7. Spano, F.C. The Spectral Signatures of Frenkel Polarons in H- and J-Aggregates. Acc. Chem. Res. 2010, 43, 429-439. [CrossRef]

8. Hestand, N.J.; Spano, F.C. Molecular Aggregate Photophysics beyond the Kasha Model: Novel Design Principles for Organic Materials. Acc. Chem. Res. 2017, 50, 341-350. [CrossRef]

9. Hestand, N.J.; Spano, F.C. Expanded Theory of H- and J-Molecular Aggregates: The Effects of Vibronic Coupling and Intermolecular Charge Transfer. Chem. Rev. 2018, 118, 7069-7163. [CrossRef]

10. Yu, P.; Zhen, Y.; Dong, H.; Hu, W. Crystal Engineering of Organic Optoelectronic Materials. Chem 2019, 5, 2814-2853. [CrossRef]

11. Würthner, F.; Saha-Möller, C.R.; Fimmel, B.; Ogi, S.; Leowanawat, P.; Schmidt, D. Perylene Bisimide Dye Assemblies as Archetype Functional Supramolecular Materials. Chem. Rev. 2016, 116, 962-1052. [CrossRef] [PubMed]

12. Hecht, M.; Würthner, F. Supramolecularly Engineered J-Aggregates Based on Perylene Bisimide Dyes. Acc. Chem. Res. 2021, 54, 642-653. [CrossRef] [PubMed]

13. Hoeben, F.J.M.; Jonkheijm, P.; Meijer, E.W.; Schenning, A.P.H.J. About supramolecular assemblies of pi-conjugated systems. Chem. Rev. 2005, 105, 1491-1546. [CrossRef] [PubMed]

14. Su, F.; Chen, G.; Korevaar, P.A.; Pan, F.; Liu, H.; Guo, Z.; Schenning, A.P.H.J.; Zhang, H.; Lin, J.; Jiang, Y. Discrete $\pi$-Stacks from Self-Assembled Perylenediimide Analogues. Angew. Chem. Int. Ed. Engl. 2019, 58, 15273-15277. [CrossRef] [PubMed]

15. Yang, L.; Langer, P.; Davies, E.S.; Baldoni, M.; Wickham, K.; Besley, N.A.; Besley, E.; Champness, N.R. Synthesis and characterisation of rylene diimide dimers using molecular handcuffs. Chem. Sci. 2019, 10, 3723-3732. [CrossRef] [PubMed]

16. Kaufmann, C.; Bialas, D.; Stolte, M.; Würthner, F. Discrete $\pi$-Stacks of Perylene Bisimide Dyes within Folda-Dimers: Insight into Long- and Short-Range Exciton Coupling. J. Am. Chem. Soc. 2018, 140, 9986-9995. [CrossRef]

17. Kaufmann, C.; Kim, W.; Nowak-Król, A.; Hong, Y.; Kim, D.; Würthner, F. Ultrafast Exciton Delocalization, Localization, and Excimer Formation Dynamics in a Highly Defined Perylene Bisimide Quadruple $\pi$-Stack. J. Am. Chem. Soc. 2018, 140, 4253-4258. [CrossRef]

18. Bardeen, C.J. The Structure and Dynamics of Molecular Excitons. Annu. Rev. Phys. Chem. 2014, 65, 127-148. [CrossRef]

19. Mewes, S.A.; Dreuw, A. Density-based descriptors and exciton analyses for visualizing and understanding the electronic structure of excited states. Phys. Chem. Chem. Phys. 2019, 21, 2843-2856. [CrossRef]

20. Darghouth, A.A.M.H.M.; Correa, G.C.; Juillard, S.; Casida, M.E.; Humeniuk, A.; Mitrić, R. Davydov-type excitonic effects on the absorption spectra of parallel-stacked and herringbone aggregates of pentacene: Time-dependent density-functional theory and time-dependent density-functional tight binding. J. Chem. Phys. 2018, 149, 134111. [CrossRef]

21. Casanova, D. Theoretical investigations of the perylene electronic structure: Monomer, dimers, and excimers. Int. J. Quantum Chem. 2015, 115, 442-452. [CrossRef]

22. Zubiria-Ulacia, M.; Matxain, J.M.; Casanova, D. The role of CT excitations in PDI aggregates. Phys. Chem. Chem. Phys. 2020, 22, 15908-15918. [CrossRef] [PubMed]

23. Mao, Y.; Montoya-Castillo, A.; Markland, T.E. Accurate and efficient DFT-based diabatization for hole and electron transfer using absolutely localized molecular orbitals. J. Chem. Phys. 2019, 151, 164114. [CrossRef] [PubMed]

24. Mao, Y.; Montoya-Castillo, A.; Markland, T.E. Excited state diabatization on the cheap using DFT: Photoinduced electron and hole transfer. J. Chem. Phys. 2020, 153, 244111. [CrossRef] [PubMed]

25. Mewes, S.A.; Plasser, F.; Krylov, A.; Dreuw, A. Benchmarking Excited-State Calculations Using Exciton Properties. J. Chem. Theory Comput. 2018, 14, 710-725. [CrossRef] [PubMed]

26. Jurinovich, S.; Cupellini, L.; Guido, C.A.; Mennucci, B. EXAT: EXcitonic analysis tool. J. Comput. Chem. 2018, 39, 279-286. [CrossRef]

27. Walter, C.; Krämer, V.; Engels, B. On the applicability of time-dependent density functional theory (TDDFT) and semiempirical methods to the computation of excited-state potential energy surfaces of perylene-based dye-aggregates. Int. J. Quantum Chem. 2017, 117, e25337. [CrossRef]

28. Accomasso, D.; Persico, M.; Granucci, G. Diabatization by Localization in the Framework of Configuration Interaction Based on Floating Occupation Molecular Orbitals (FOMO-CI). ChemPhotoChem 2019, 3, 933-944. [CrossRef]

29. Tamura, H. Diabatization for Time-Dependent Density Functional Theory: Exciton Transfers and Related Conical Intersections. J. Phys. Chem. A 2016, 120, 9341-9347. [CrossRef]

30. Liu, W.; Lunkenheimer, B.; Settels, V.; Engels, B.; Fink, R.F.; Köhn, A. A general ansatz for constructing quasi-diabatic states in electronically excited aggregated systems. J. Chem. Phys. 2015, 143, 084106. [CrossRef]

31. Carreras, A.; Uranga-Barandiaran, O.; Castet, F.; Casanova, D. Photophysics of Molecular Aggregates from Excited State Diabatization. J. Chem. Theory Comput. 2019, 15, 2320-2330. [CrossRef] [PubMed]

32. Shirai, S.; Iwata, S.; Tani, T.; Inagaki, S. Ab Initio Studies of Aromatic Excimers Using Multiconfiguration Quasi-Degenerate Perturbation Theory. J. Phys. Chem. A 2011, 115, 7687-7699. [CrossRef] [PubMed] 
33. Lv, M.; Lu, X.; Jiang, Y.; Sandoval-Salinas, M.E.; Casanova, D.; Sun, H.; Sun, Z.; Xu, J.; Yang, Y.; Chen, J. Near-Unity Triplet Generation Promoted via Spiro-Conjugation. Angew. Chemie Int. Ed. 2021, 61, 2-10. [CrossRef]

34. Margulies, E.A.; Logsdon, J.L.; Miller, C.E.; Ma, L.; Simonoff, E.; Young, R.M.; Schatz, G.C.; Wasielewski, M.R. Direct Observation of a Charge-Transfer State Preceding High-Yield Singlet Fission in Terrylenediimide Thin Films. J. Am. Chem. Soc. 2017, 139, 663-671. [CrossRef]

35. Margulies, E.A.; Miller, C.E.; Wu, Y.; Ma, L.; Schatz, G.C.; Young, R.M.; Wasielewski, M.R. Enabling singlet fission by controlling intramolecular charge transfer in $\pi$-stacked covalent terrylenediimide dimers. Nat. Chem. 2016, 8, 1120-1125. [CrossRef]

36. Young, R.M.; Wasielewski, M.R. Mixed Electronic States in Molecular Dimers: Connecting Singlet Fission, Excimer Formation, and Symmetry-Breaking Charge Transfer. Acc. Chem. Res. 2020, 53, 1957-1968. [CrossRef] [PubMed]

37. Beljonne, D.; Yamagata, H.; Brédas, J.L.; Spano, F.C.; Olivier, Y. Charge-Transfer Excitations Steer the Davydov Splitting and Mediate Singlet Exciton Fission in Pentacene. Phys. Rev. Lett. 2013, 110, 226402. [CrossRef]

38. Berkelbach, T.C.; Hybertsen, M.S.; Reichman, D.R. Microscopic theory of singlet exciton fission. II. Application to pentacene dimers and the role of superexchange. J. Chem. Phys. 2013, 138, 114103. [CrossRef]

39. Zeng, T.; Hoffmann, R.; Ananth, N. The Low-Lying Electronic States of Pentacene and Their Roles in Singlet Fission. J. Am. Chem. Soc. 2014, 136, 5755-5764. [CrossRef]

40. Casanova, D. Theoretical Modeling of Singlet Fission. Chem. Rev. 2018, 118, 7164-7207. [CrossRef]

41. Han, G.; Yi, Y. Local Excitation/Charge-Transfer Hybridization Simultaneously Promotes Charge Generation and Reduces Nonradiative Voltage Loss in Nonfullerene Organic Solar Cells. J. Phys. Chem. Lett. 2019, 10, 2911-2918. [CrossRef] [PubMed]

42. Coropceanu, V.; Chen, X.-K.; Wang, T.; Zheng, Z.; Brédas, J.-L. Charge-transfer electronic states in organic solar cells. Nat. Rev. Mater. 2019, 4, 689-707. [CrossRef]

43. Dong, Y.; Nikolis, V.C.; Talnack, F.; Chin, Y.-C.; Benduhn, J.; Londi, G.; Kublitski, J.; Zheng, X.; Mannsfeld, S.C.B.; Spoltore, D.; et al Orientation dependent molecular electrostatics drives efficient charge generation in homojunction organic solar cells. Nat. Commun. 2020, 11, 4617. [CrossRef] [PubMed]

44. Shi, B.; Nachtigallová, D.; Aquino, A.J.A.; Machado, F.B.C.; Lischka, H. Excited states and excitonic interactions in prototypic polycyclic aromatic hydrocarbon dimers as models for graphitic interactions in carbon dots. Phys. Chem. Chem. Phys. 2019, 21,9077-9088. [CrossRef] [PubMed]

45. Fliegl, H.; You, Z.; Hsu, C.; Sundholm, D. The Excitation Spectra of Naphthalene Dimers: Frenkel and Charge-transfer Excitons. J. Chin. Chem. Soc. 2016, 63, 20-32. [CrossRef]

46. Liu, W.; Settels, V.; Harbach, P.H.P.; Dreuw, A.; Fink, R.F.; Engels, B. Assessment of TD-DFT- and TD-HF-based approaches for the prediction of exciton coupling parameters, potential energy curves, and electronic characters of electronically excited aggregates J. Comput. Chem. 2011, 32, 1971-1981. [CrossRef]

47. Settels, V.; Liu, W.; Pflaum, J.; Fink, R.F.; Engels, B. Comparison of the electronic structure of different perylene-based dyeaggregates. J. Comput. Chem. 2012, 33, 1544-1553. [CrossRef]

48. Mukazhanova, A.; Malone, W.; Negrin-Yuvero, H.; Fernandez-Alberti, S.; Tretiak, S.; Sharifzadeh, S. Photoexcitation dynamics in perylene diimide dimers. J. Chem. Phys. 2020, 153, 244117. [CrossRef]

49. Bellinger, D.; Pflaum, J.; Brüning, C.; Engel, V.; Engels, B. The electronic character of PTCDA thin films in comparison to other perylene-based organic semi-conductors: Ab initio-, TD-DFT and semi-empirical computations of the opto-electronic properties of large aggregates. Phys. Chem. Chem. Phys. 2017, 19, 2434-2448. [CrossRef]

50. Engels, B.; Engel, V. The dimer-approach to characterize opto-electronic properties of and exciton trapping and diffusion in organic semiconductor aggregates and crystals. Phys. Chem. Chem. Phys. 2017, 19, 12604-12619. [CrossRef]

51. Hartzler, D.A.; Slipchenko, L.V.; Savikhin, S. Triplet-Triplet Coupling in Chromophore Dimers: Theory and Experiment. J. Phys. Chem. A 2018, 122, 6713-6723. [CrossRef] [PubMed]

52. You, Z.-Q.; Hsu, C.-P.; Fleming, G.R. Triplet-triplet energy-transfer coupling: Theory and calculation. J. Chem. Phys. 2006, 124, 044506. [CrossRef] [PubMed]

53. Gillett, A.J.; Privitera, A.; Dilmurat, R.; Karki, A.; Qian, D.; Pershin, A.; Londi, G.; Myers, W.K.; Lee, J.; Yuan, J.; et al. The role of charge recombination to triplet excitons in organic solar cells. Nature 2021, 597, 666-671. [CrossRef] [PubMed]

54. Bassan, E.; Gualandi, A.; Cozzi, P.G.; Ceroni, P. Design of BODIPY dyes as triplet photosensitizers: Electronic properties tailored for solar energy conversion, photoredox catalysis and photodynamic therapy. Chem. Sci. 2021, 12, 6607-6628. [CrossRef]

55. Gibbons, D.J.; Farawar, A.; Mazzella, P.; Leroy-Lhez, S.; Williams, R.M. Making triplets from photo-generated charges: Observations, mechanisms and theory. Photochem. Photobiol. Sci. 2020, 19, 136-158. [CrossRef]

56. Cakmak, Y.; Kolemen, S.; Duman, S.; Dede, Y.; Dolen, Y.; Kilic, B.; Kostereli, Z.; Yildirim, L.T.; Dogan, A.L.; Guc, D.; et al. Designing Excited States: Theory-Guided Access to Efficient Photosensitizers for Photodynamic Action. Angew. Chem. Int. Ed. 2011, 50, 11937-11941. [CrossRef]

57. Galán, L.A.; Andrés Castán, J.M.; Dalinot, C.; Marqués, P.S.; Galiana, J.; Blanchard, P.; Andraud, C.; Dumont, E.; Maury, O.; Cabanetos, C.; et al. Exploring the Concept of Dimerization-Induced Intersystem Crossing: At the Origins of Spin-Orbit Coupling Selection Rules. J. Phys. Chem. B 2021, 125, 8572-8580. [CrossRef]

58. Oleson, A.; Zhu, T.; Dunn, I.S.; Bialas, D.; Bai, Y.; Zhang, W.; Dai, M.; Reichman, D.R.; Tempelaar, R.; Huang, L.; et al. Perylene Diimide-Based Hj- and hJ-Aggregates: The Prospect of Exciton Band Shape Engineering in Organic Materials. J. Phys. Chem. C 2019, 123, 20567-20578. [CrossRef] 
59. Liu, W.; Canola, S.; Köhn, A.; Engels, B.; Negri, F.; Fink, R.F. A model hamiltonian tuned toward high level ab initio calculations to describe the character of excitonic states in perylenebisimide aggregates. J. Comput. Chem. 2018, 39, 1979-1989. [CrossRef]

60. Canola, S.; Bagnara, G.; Dai, Y.; Ricci, G.; Calzolari, A.; Negri, F. Addressing the Frenkel and charge transfer character of exciton states with a model Hamiltonian based on dimer calculations: Application to large aggregates of perylene bisimide. J. Chem. Phys. 2021, 154, 124101. [CrossRef]

61. Hellweg, A.; Grün, S.A.; Hättig, C. Benchmarking the performance of spin-component scaled CC2 in ground and electronically excited states. Phys. Chem. Chem. Phys. 2008, 10, 4119. [CrossRef] [PubMed]

62. Winter, N.O.C.; Graf, N.K.; Leutwyler, S.; Hättig, C. Benchmarks for 0-0 transitions of aromatic organic molecules: DFT/B3LYP, ADC(2), CC2, SOS-CC2 and SCS-CC2 compared to high-resolution gas-phase data. Phys. Chem. Chem. Phys. 2013, 15, 6623-6630. [CrossRef] [PubMed]

63. Dreuw, A.; Wormit, M. The algebraic diagrammatic construction scheme for the polarization propagator for the calculation of excited states. Wiley Interdiscip. Rev. Comput. Mol. Sci. 2015, 5, 82-95. [CrossRef]

64. Plasser, F.; Lischka, H. Analysis of Excitonic and Charge Transfer Interactions from Quantum Chemical Calculations. J. Chem. Theory Comput. 2012, 8, 2777-2789. [CrossRef] [PubMed]

65. Casanova, D.; Krylov, A.I. Quantifying local exciton, charge resonance, and multiexciton character in correlated wave functions of multichromophoric systems. J. Chem. Phys. 2016, 144, 014102. [CrossRef] [PubMed]

66. Plasser, F. TheoDORE: A toolbox for a detailed and automated analysis of electronic excited state computations. J. Chem. Phys. 2020, 152, 084108. [CrossRef] [PubMed]

67. Petelenz, P. Mixing of Frenkel excitons with charge transfer states in the neighbourhood of charged defect. Chem. Phys. Lett. 1977, 47, 603-605. [CrossRef]

68. Norton, J.E.; Brédas, J.-L. Theoretical characterization of titanyl phthalocyanine as a p-type organic semiconductor: Short intermolecular $\pi-\pi$ interactions yield large electronic couplings and hole transport bandwidths. J. Chem. Phys. 2008, 128, 034701. [CrossRef]

69. Kim, D. A Theoretical Analysis of the Excited State of Oligoacene Aggregates: Local Excitation vs. Charge-Transfer Transition. Bull. Korean Chem. Soc. 2015, 36, 2284-2289. [CrossRef]

70. Löwdin, P. On the Non-Orthogonality Problem Connected with the Use of Atomic Wave Functions in the Theory of Molecules and Crystals. J. Chem. Phys. 1950, 18, 365-375. [CrossRef]

71. Nottoli, M.; Jurinovich, S.; Cupellini, L.; Gardiner, A.T.; Cogdell, R.; Mennucci, B. The role of charge-transfer states in the spectral tuning of antenna complexes of purple bacteria. Photosynth. Res. 2018, 137, 215-226. [CrossRef]

72. Fink, R.F.; Seibt, J.; Engel, V.; Renz, M.; Kaupp, M.; Lochbrunner, S.; Zhao, H.-M.; Pfister, J.; Würthner, F.; Engels, B. Exciton trapping in pi-conjugated materials: A quantum-chemistry-based protocol applied to perylene bisimide dye aggregates. J. Am. Chem. Soc. 2008, 130, 12858-12859. [CrossRef] [PubMed]

73. Hirata, S.; Head-Gordon, M. Time-dependent density functional theory within the Tamm-Dancoff approximation. Chem. Phys. Lett. 1999, 314, 291-299. [CrossRef]

74. Yanai, T.; Tew, D.P.; Handy, N.C. A new hybrid exchange-correlation functional using the Coulomb-attenuating method (CAM-B3LYP). Chem. Phys. Lett. 2004, 393, 51-57. [CrossRef]

75. Chai, J.-D.; Head-Gordon, M. Long-range corrected hybrid density functionals with damped atom-atom dispersion corrections. Phys. Chem. Chem. Phys. 2008, 10, 6615. [CrossRef]

76. Frisch, M.J.; Trucks, G.W.; Schlegel, H.B.; Scuseria, G.E.; Robb, M.A.; Cheeseman, J.R.; Scalmani, G.; Barone, V.; Petersson, G.A.; Nakatsuji, H.; et al. Gaussian 16; Gaussian, Inc.: Wallingford, CT, USA, 2016.

77. Hestand, N.J.; Kazantsev, R.V.; Weingarten, A.S.; Palmer, L.C.; Stupp, S.I.; Spano, F.C. Extended-Charge-Transfer Excitons in Crystalline Supramolecular Photocatalytic Scaffolds. J. Am. Chem. Soc. 2016, 138, 11762-11774. [CrossRef]

78. Stehr, V.; Engels, B.; Deibel, C.; Fink, R.F. Anisotropy of singlet exciton diffusion in organic semiconductor crystals from ab initio approaches. J. Chem. Phys. 2014, 140, 024503. [CrossRef] 